\title{
Effect of different levels of drought stress on the germination and seedling growth parameters of three wheat cullivars seeds
}

\author{
Abdalla M. A. Mansour* \\ Department of Plant Production, \\ Faculty of Agriculture, \\ University of Benghazi. Libya
}

\section{Twfeik Elbagrmi}

Department of Environmental Health, Faculty of public health, University of Benghazi. Libya

Abdalla.mansour@uob.edu.ly

https://doi.org/10.36602/jmuas.2019.v01.01.22

\begin{abstract}
Seed germination of three varieties of Wheat (Triticum aestivum L.) CONDOR, SERI and T.R. evaluated under 5 drought levels $(0.0 \mathrm{mPa}$ (control), $-0.3 \mathrm{mPa},-0.6 \mathrm{mPa},-0.9 \mathrm{mPa}$ and $-1.2 \mathrm{mPa}$ ). We used polyethylene glycol (PEG6000) to make drought stress conditions. Germinated seeds were counted daily up to 14 days under laboratory conditions.

Percentage and mean germination time, coefficients of germination, mean germination rate, and uncertainty of germination process as germination parameters. Shoots and roots length; fresh, dry weight of shoots and roots, leaves and roots number, surface area of leaves as seedling growth parameters were studied. The data was analyzed statistically using SPSS software, to identify significant difference among wheat varieties and among treatment.

Comparison between means showed that the highest value for most of germination parameters recorded for SERI variety followed by T.R., and most of seedling growth parameters were recorded for T.R. followed by SERI. The results determined that seeds of all varieties germinate well in the lowest concentration of PEG. Increase the concentration of PEG affect all germination and seedling growth parameters.
\end{abstract}

Key words: drought stress- germination- wheat- PEG 6000- Triticum aestivum

\section{Introduction}

Wheat (Triticum aestivum L.) is a staple food for more than $35 \%$ of the world population and it is also the first grain crops in most of developing countries(Metwali, et al., 2011),(Jing and Chang 2003) . Bread wheat is the main food of people in many countries and about $70 \%$ calories and $80 \%$ protein of human is supplied from its consumption(Taregh, et al., 2011). drought stress is one of the major causes of crop loss worldwide, which commonly reduces average yield for many crop plants by more than 50\% (Wang, et al., 2003); ( Bayoumi, et al., 2008).Depending on which stage of growth a plant experiences drought stress, it reacts quite differently to the stress. Plant affected 
by drought at any time, stage such as germination and seedling growth are critical (Pessarakli 1999). With progressive global climatic change and increasing shortage of water resources and worsening eco-environment, wheat production is influenced greatly (Khan and Naqvi 2011).

Drought stress, which is the most serious environmental problem limiting crop production in rain-fed agriculture (Bahieldin et al., 2005), can severely impact plant growth and development, limit plant production and the crop performance(Shao et al., 2009). Drought is a major abiotic factor that limits agricultural crop production. Drought stress affects 40 to $60 \%$ of the world's agriculture lands (Shahryari and Mollasadeghi 2011) seriously constraining global crop production(pan et al., 2002). Germination is the first critical phase most affected by drought (Ashraf and Mehmood 1990).

Dhandas et al., indicated that seed vigor index and shoot length are among the most sensitive to drought stress, followed by root length and coleoptiles length(Dhanda, et al., 2004). The rate of seed germination and the final germination percentage as well as the amount of water absorbed by the seeds were considerably lowered with the rise of osmotic stress level (Heikal, et al., 1981). Selection of drought tolerance at early seedling stage is frequently accomplished using simulated drought induced by chemicals like polyethylene glycol (PEG6000) under laboratory conditions.

Previous studies revealed that PEG can be used to modify the osmotic potential of nutrient solution culture and thus induce plant water deficit in a relatively controlled manner (Money, 1989); ( Zhu, 2006). Lu and Neumann; Kulkarni and Deshpande Showed that PEG molecules are inert, no-ionic, virtually impermeable to cell membranes and can induce uniform water stress without causing direct physiological damage. (Lu and Neumann, 1998); (Kulkarni and Deshpande, 2007) PEG as a factor causing drought stress by reducing water potential results in reducing growth in seed germinated and stopping seedling growth so that this effect has been observed more in the shoot than primary roots (Zhu, 2006). Dodd and Donovan also suggested that PEG prevent water absorption by seeds, but penetrable ions by reducing potential inside cell results in water absorption and starting to germinate (Dodd and Donovan, 1999).

Development of stress tolerant varieties is always a main objective of many breeding programs, but success has been limited by adequate screening techniques, and the lack of genotypes that show clear differences in response to various environmental stresses. Therefore, wheat breeders are always looking for means and sources of genetic improvement for grain yield and other agronomic traits. Plant breeders are also adopting new technologies such as molecular markers to increase wheat grain yield under drought stress regions (Khakwani,et al.,2011). Agriculture has been affected by environmental stresses such as drought, salinity, extreme temperatures, chemical toxicity and oxidative stress which reduce crop yield fifty percent, approximately and water stress that is caused 
by salinity and drought is a prevalent problem in the world. However, plants are affected by drought and salinity similarly (Khayatnezhad, et al., 2010).

Salinity and drought stresses are physiologically related, because both induce osmotic stress and most of the metabolic responses of the affected plants are similar to some extent (Kumar, et al., 2011) such as seed soaking in solutions of Polyethylene Glycol (PEG) was expressed as sowing seeds in an osmotic solution that permits seed to absorb water for germination, but inhibits radicle extension via seed coat (Janmohammadi and Sharifzadeh, 2008);(Giri and Schillinger, 2003). The reaction of seed priming is associated with some factors including duration of priming, seed maturity, species and environmental conditions (Armin, et al., 2010).

Since there are differences between species and even different varieties in terms of susceptibility to drought stress, the aim of this study is to investigate the effects of drought stress on germination seedling stage of three wheat genotype. The present study was conduct to evaluate three wheat cultivars for drought resistance at germination and seedling stage. PEG-6000 was used as an osmotic to induce stress conditions.

\section{Materials and methods}

In order to study the effects of drought stress, using polyethylene glycol, on germination and seedling growth parameters in wheat, experiment was conducted in Department of Plant Production, Faculty of Agriculture-Soluq Benghazi University, Libya in 2015. The form of experiment was factorial, completely randomized design with five replications. Seeds of three wheat cultivars (Condur, Seri, and T.R.) were used. These cultivars were obtained from al-kufra Agriculture project in Libya, where all obtained from CIMMYT.

Wheat seeds were subjected to five stress level of polyethylene glycol (PEG6000) (ACROS, company) (0.0 mPa (control), $-0.3 \mathrm{mPa},-0.6 \mathrm{mPa},-0.9 \mathrm{mPa}$ and $-1.2 \mathrm{mPa}$ ) prepared according Michel and Kaufmann (Kaufmann and Eckard, 1971);(Michel and Kaufmann, 1973) PEG 6000 was prepared by dissolving the required amount of PEG6000 in distilled water at $25^{\circ} \mathrm{C}$. Wheat grains were disinfected with $5 \%$ sodium hypochlorite $(\mathrm{NaOCl})$ solution for 3 minutes. Then seeds were washed three times with distilled water. 20 grains from each cultivars were germinated on two layers of filter paper in plastic Petri dishes filter papers were moistened with respective treatment from PEG6000. Petri dishes were covered and sealed with parafilm to prevent evaporation of moisture (Emmerich and Hardegree, 1991) and incubated under laboratory condition $\left(27 \pm 2{ }^{\circ} \mathrm{C}\right)$ for 14 days.

Seeds were considered germinated when exhibited radicle extension of $>2 \mathrm{~mm}$. Every 24 hours germinated seeds were counted daily during the course of the experiment to determine following germination parameters. [Germination percentage $(G \%)$, mean germination time (MGT), Coefficient of variation $(\mathrm{CVi})$, mean germination rate (MGR) 
and Uncertainty of Germination Process (GU)]. (ISTA, 1993 \& 1999); (Sadeghi et al., 2011); (AOSA, 1983); (Scott, et al., 1984). All of these calculation organized in Excel sheet according to Ranal et al. (Ranal and Santana, 2006)\& (Ranal et al., 2009).

By harvest seedlings, the experiment was termined 14 days after seeds soaking and traits including [shoot length, root length, root number, leaf number, leaf surface area, seedling fresh weight, shoot fresh weight, root fresh weight, shoot dry weight, and root dry weight] were measured, (Almaghrabi, 2012).

\section{Experimental design and statistical analysis:}

Effects of two factors were analyzed in these experiments and a completely randomized design with five replications of 20 seeds per replicate. The first factor (Wheat cultivars) had three levels (Condur, Seri and T.R.), the second one (Osmotic potential - Drought levels) had five levels $(0.0,-0.3,-0.6,-0.9$ and $-1.2 \mathrm{MPa})$. The data was analyzed statistically using IBM SPSS statistics software ver. 23, 2015). Analyses of variance (ANOVA) of the obtained data were applied to identify significant difference among
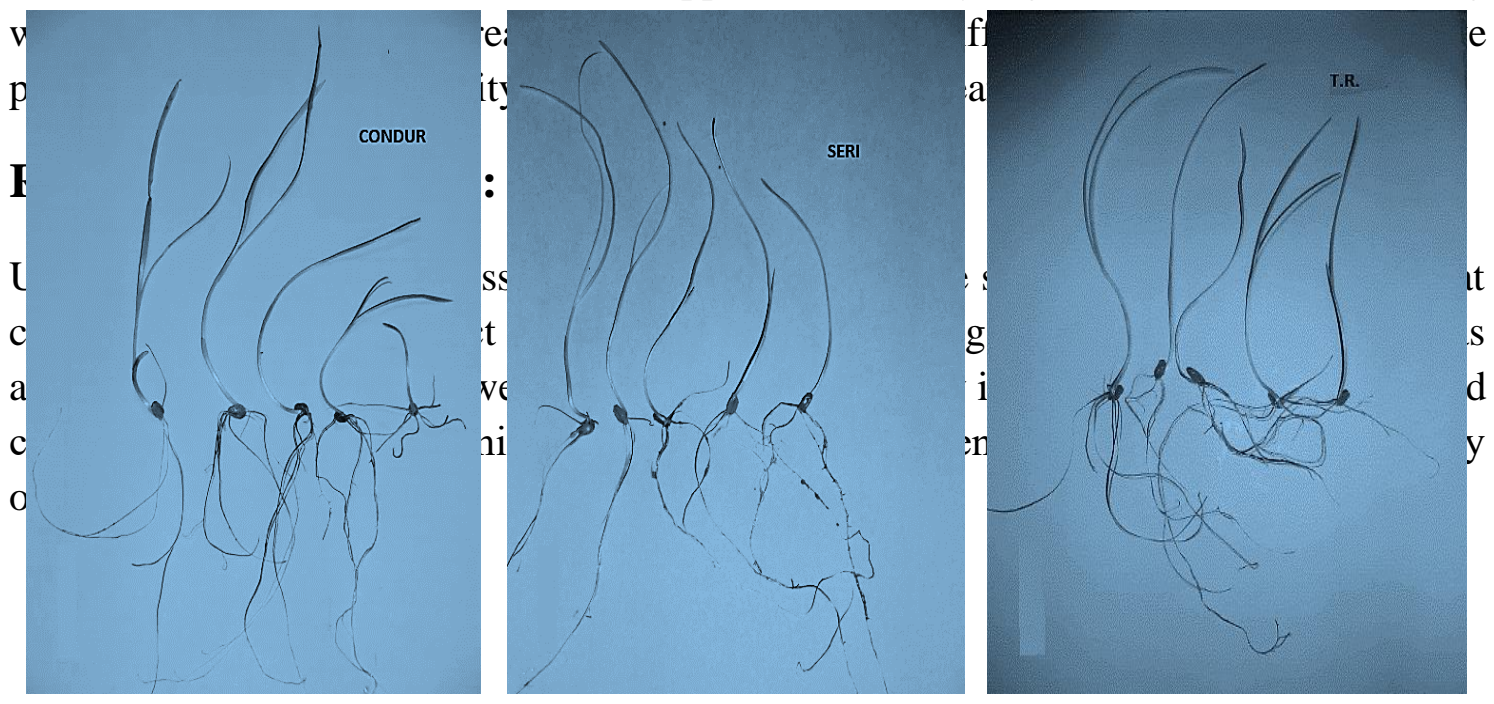

Figure 1: Effect of Different Concentration of PEG ( 5 treatments from left to right $(0.0,-0.3,-0.6,-0.9$ and $-1.2 \mathrm{MPa})$ ) on Germination of Three Wheat Cultivars (Condur, Seri and T.R.)

Table (1) show the analysis of variance for the effect of cultivars and drought levels on these parameters of wheat cultivars. From table (1) we can observe no significant differences between mean germination time (MGT) and mean germination rate (MGR). MGT indicate the time for the first germination of the faster seed germinated. 
Table 1: Analysis of variance for effect of cultivars and drought levels on germination parameters of three wheat cultivars.

\begin{tabular}{|c|c|c|c|c|c|c|}
\hline \multirow[b]{2}{*}{$\begin{array}{l}\text { Source of } \\
\text { variance }\end{array}$} & \multirow[b]{2}{*}{$D f$. } & \multicolumn{5}{|c|}{ Mean Square } \\
\hline & & 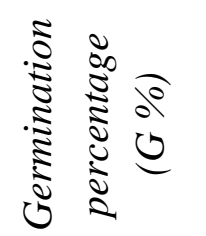 & 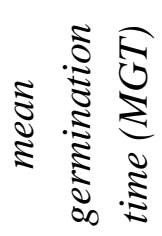 & 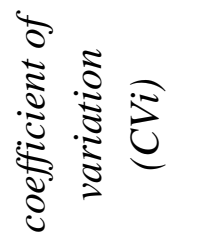 & 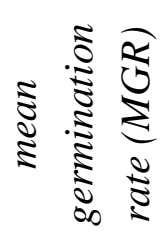 & 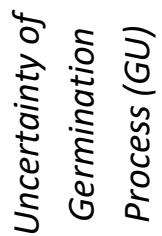 \\
\hline Cultivars & 2 & $1605.33^{* *}$ & $1.880^{n s}$ & $1639.644^{\text {** }}$ & $0.013^{n s}$ & $4.168^{* *}$ \\
\hline $\begin{array}{c}\text { Drought } \\
\text { Levels }\end{array}$ & 4 & $3795.833^{* * *}$ & $1.600^{n s}$ & $2343.889^{\text {** }}$ & $0.002^{n s}$ & $1.145^{* *}$ \\
\hline \begin{tabular}{c}
\multicolumn{2}{c}{ Cultivars } \\
$X \quad$ Drought \\
Levels
\end{tabular} & 8 & $272.83^{* *}$ & $0.466^{n s}$ & $497.919^{* *}$ & $0.004^{n s}$ & $0.495^{* *}$ \\
\hline Error & 60 & 61.167 & .975 & 194.496 & 0.005 & 0.225 \\
\hline Total & 74 & & & & & \\
\hline
\end{tabular}

Df: degree of freedom and **: significant at $5 \%$. ns: nonsignificant

Table (2) showed that the faster time for emerging of seed was 3.76, 4.12 and 4.51 day for Condur variety followed by Seri and T.R. the treatments do not showed any difference effect on mean germination time (no significant difference between treatment). In all cultivars the final germination percentage was decrease with drought stress increase, the highest germination at control and started to decrease after that. Condur cultivar had higher final germination percentage regarding of drought stress. Drought stress at germination stage can results in delayed and reduced germination.

No significant difference between varieties and treatments on mean germination time (MGT), on the base of results the effect of cultivars and treatments on the Coefficient of Variation CVi (coefficient germination time) was significant and SERI gave higher coefficient of variation followed by T.R.; high germination time was observed at -0.3 $m P a$ with 4.47 days. All germination parameters indicate that stress factor like PEG negatively affect plant growth.

According to ANOVA (table 3), PEG levels (drought levels) were significant for all the traits except for root number. The difference between the cultivars was statistically significant for all the traits except for leaf number. There was significance in the interaction between cultivars and drought levels for all parameters except root number, root fresh weight and leaf surface area. 
عدد خاص بالأوراق العلمية المقدمة للمؤتمر العلمي الأول للعلوم الزراعية - إنتاج نباتي (5-6 أكتوبر 2019)

Table 2: Effect of different drought levels on germination parameters

\begin{tabular}{|c|c|c|c|c|c|c|}
\hline parameters & $\begin{array}{c}\text { drought } \\
\text { level }\end{array}$ & Condur & Seri & T.R. & Mean & $L S D$ \\
\hline \multirow{3}{*}{$\begin{array}{c}\text { Germination } \\
\text { Percentage \% }\end{array}$} & $0.0 \mathrm{mPa}$ & 83.00 & 69.00 & 84.00 & $78.67 a$ & \multirow{5}{*}{5.715} \\
\hline & $-0.3 m P a$ & 81.00 & 63.00 & 64.00 & $69.33 b$ & \\
\hline & $-0.6 \mathrm{mPa}$ & 70.00 & 56.00 & 58.00 & $61.33 c$ & \\
\hline \multirow{4}{*}{$(G \%)$} & $-0.9 \mathrm{mPa}$ & 60.00 & 52.00 & 33.00 & $48.33 d$ & \\
\hline & $-1.2 \mathrm{mPa}$ & 48.00 & 42.00 & 27.00 & $39.00 e$ & \\
\hline & Mean & $68.40 \mathrm{~A}$ & $56.40 \mathrm{~B}$ & $53.20 \mathrm{~B}$ & & \multirow{7}{*}{0.721} \\
\hline & $L S D$ & \multicolumn{4}{|c|}{4.42} & \\
\hline \multirow{7}{*}{$\begin{array}{c}\text { Mean Germination } \\
\text { Time (day) }\end{array}$} & $0.0 \mathrm{mPa}$ & 3.3900 & 4.1220 & 4.6420 & $4.05 a b$ & \\
\hline & $-0.3 \mathrm{mPa}$ & 4.5940 & 4.4120 & 4.4180 & $4.47 a$ & \\
\hline & $-0.6 \mathrm{mPa}$ & 4.3240 & 4.4840 & 4.4560 & $4.42 a b$ & \\
\hline & $-0.9 m P a$ & 3.9060 & 3.9500 & 4.4420 & $4.10 a b$ & \\
\hline & $-1.2 m P a$ & 3.2000 & 3.6060 & 4.1880 & $3.67 b$ & \\
\hline & Mean & $3.8828 \mathrm{~A}$ & $4.1148 \mathrm{~A}$ & $4.4292 A$ & & \\
\hline & $L S D$ & \multicolumn{4}{|c|}{0.559} & \\
\hline \multirow{7}{*}{$\begin{array}{c}\text { Coefficient of } \\
\text { Variation } \\
\text { (coefficient } \\
\text { germination } \\
\text { time)\% }\end{array}$} & $0.0 \mathrm{mPa}$ & 39.2800 & 54.7060 & 46.1420 & $46.71 b$ & \multirow{5}{*}{10.144} \\
\hline & $-0.3 m P a$ & 52.2820 & 55.7680 & 71.0520 & $59.70 a$ & \\
\hline & $-0.6 \mathrm{mPa}$ & 43.1520 & 67.1500 & 40.9380 & $50.41 a b$ & \\
\hline & $-0.9 \mathrm{mPa}$ & 42.5820 & 48.1940 & 32.4540 & $41.08 \mathrm{~b}$ & \\
\hline & $-1.2 \mathrm{mPa}$ & 6.6660 & 39.1060 & 34.1840 & $25.99 c$ & \\
\hline & Mean & $36.7924 B$ & $52.9848 \mathrm{~A}$ & $44.5540 B$ & & \\
\hline & $L S D$ & \multicolumn{4}{|c|}{7.857} & \\
\hline \multirow{3}{*}{$\begin{array}{c}\text { Mean Germination } \\
\text { Rate }\left(\text { day }^{-1}\right)\end{array}$} & $0.0 \mathrm{mPa}$ & 0.3264 & 0.2520 & 0.2208 & $0.266 a$ & \multirow{3}{*}{0.0539} \\
\hline & $-0.3 m P a$ & 0.2344 & 0.2378 & 0.2284 & $0.233 a$ & \\
\hline & $-0.6 \mathrm{mPa}$ & 0.2880 & 0.2252 & 0.2472 & $0.253 a$ & \\
\hline
\end{tabular}




\begin{tabular}{|c|c|c|c|c|c|c|}
\hline & $-0.9 \mathrm{mPa}$ & 0.2668 & 0.2572 & 0.2092 & $0.244 a$ & \\
\hline & $-1.2 \mathrm{mPa}$ & 0.2400 & 0.2852 & 0.2242 & $0.250 a$ & \\
\hline & Mean & $0.2711 \mathrm{~A}$ & $0.2515 A B$ & $0.2260 B$ & & \\
\hline & $L S D$ & \multicolumn{4}{|c|}{0.0417} & \\
\hline \multirow{3}{*}{$\begin{array}{c}\text { Uncertainty of } \\
\text { Germination } \\
\text { Process }\end{array}$} & $0.0 \mathrm{mPa}$ & 1.3182 & 2.0540 & 2.3354 & $1.90 a$ & \multirow{5}{*}{0.347} \\
\hline & $-0.3 \mathrm{mPa}$ & 1.3400 & 2.0944 & 2.0976 & $1.84 a$ & \\
\hline & $-0.6 \mathrm{mPa}$ & 1.8514 & 1.9808 & 1.7684 & $1.87 a$ & \\
\hline \multirow[t]{4}{*}{ bit $(G U)$} & $-0.9 \mathrm{mPa}$ & 1.5100 & 2.1124 & 1.8430 & $1.82 a$ & \\
\hline & $-1.2 \mathrm{mPa}$ & 0.3170 & 1.8320 & 1.5850 & $1.24 b$ & \\
\hline & Mean & $1.2673 \mathrm{~B}$ & $2.0147 A$ & $1.9258 \mathrm{~A}$ & & \\
\hline & $L S D$ & \multicolumn{4}{|c|}{0.269} & \\
\hline
\end{tabular}

Values in mean column (between treatments) \& rows (between cultivars) sharing same letter are statistically no-significant at $5 \%$.

The presence of increased concentration of PEG during seedling growth inhibits the traits of this stage, maximum shoot length was recorded in T.R. (16.168 cm.) followed by Seri and Condur (15.454 and $15.166 \mathrm{~cm}$.) at level of $-0.3 \mathrm{mPa}$. Whereas the maximum root length recorded in T.R. (19.309 cm.) followed by Condur $14.506 \mathrm{~cm}$. results showed that the highest root length was at level $-0.9 \mathrm{mPa}$. Shoot length increase slightly at level of $0.3 \mathrm{mPa}$ in all cultivars and then reduced with the increase in PEG concentration. Results showed increase in root length as an adaptation with water deficient cause of PEG presence. Reduction in shoot length under drought condition result of an inhibition of cell division and elongation (Fraser, et al., 1990); (Kamran et al., 2009); (Chachar et al., 2014a);( Chachar et al., 2014b) and (Chachar et al., 2016).

For seedling and shoot fresh weight $(\mathrm{g} / \mathrm{shoot})$, the shoot fresh weight values were decreased with increasing water stress in all examined cultivars, greatest shoot fresh weight recorded in T.R. cultivar $0.522 \mathrm{~g} /$ shoot as average at control level, followed by Condur. The reduction in shoot fresh weight was attributed to lower number and development of smaller leaves with increased PEG concentration. (Chachar et al., 2014a);(Khakwani, et al., 2011).

Also the greatest root fresh weight recorded in T.R. cultivar followed by Seri 0.1068 and $0.0868 \mathrm{~g} /$ root respectively. Mean values for shoot and root dry weight in different PEG concentration were recorded for shoot $0.098,0.062,0.033,0.034$ and 0.026 and for root 
0.056, 0.026, 0.022, 0.015 and 0.011 in control, $-0.3,-0.6,-0.9$ and $-1.2 \mathrm{MPa}$ respectively, with no significant difference between drought levels up to $-0.3 \mathrm{MPa}$.

Maximum shoot dry weight of T.R. cultivar under control condition $0.224 \mathrm{~g} / \mathrm{shoot}$, minimum shoot dry weight was observed with Condur cultivar. Maximum root dry weight of T.R. cultivar under control condition $0.1360 \mathrm{~g} / \mathrm{root}$, minimum root dry weight was observed with Condur cultivar. The decrease of shoot and root dry weight was reported by (Kamran et al., 2009); (Ahmad et al., 2013); (Chachar et al., 2014a); ( Chachar et al., 2014b) and (Chachar et al., 2016) Who found that water stress had a significant effect on shoot and root dry weight. Root biomass is very important traits while selecting drought tolerant genotypes (Steven et al., 2016)

Same observation noted for leaf surface area increase of drought level reduce leaf surface area of all wheat cultivar, the maximum leaf surface area recorded with T.R. variety with $4.44 \mathrm{~cm}^{2}$ followed by Condur and Seri with 3.1734 and $3.1332 \mathrm{~cm}^{2}$ respectively at control condition. The values decrease to 50\% approximately with PEG concentration increase.

Table 3: Analysis of variance for effect of cultivars and drought levels on growth parameters of three wheat cultivars.

\begin{tabular}{|c|c|c|c|c|c|c|c|c|c|c|c|}
\hline \multirow[b]{2}{*}{$\begin{array}{l}\text { Source of } \\
\text { variance }\end{array}$} & \multirow[b]{2}{*}{$\begin{array}{l}D \\
f\end{array}$} & \multicolumn{10}{|c|}{ Mean Square } \\
\hline & & 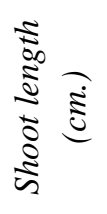 & 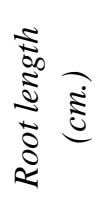 & $\begin{array}{l}\bar{\Xi} \\
\stackrel{\Xi}{\vdots} \\
\vdots \\
\vdots \\
0 \\
2\end{array}$ & 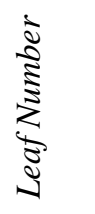 & 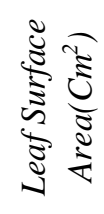 & 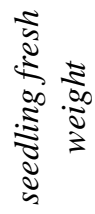 & 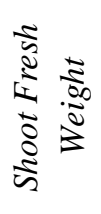 & $\begin{array}{l}\frac{2}{2} \\
\frac{5}{5} \\
\frac{5}{5} \\
\frac{5}{5}\end{array}$ & 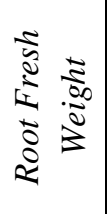 & 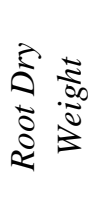 \\
\hline Cultivars & 2 & $44.739^{*}$ & $89.468^{*}$ & $1.764^{*}$ & $0.240^{n s}$ & $13.253^{*}$ & $0.007^{*}$ & $0.694^{*}$ & $0.061^{*}$ & $0.023^{*}$ & $0.011^{*}$ \\
\hline $\begin{array}{c}\text { Drought } \\
\text { Levels }\end{array}$ & 4 & $\underset{*}{128.591}$ & $\begin{array}{c}108.744 \\
*\end{array}$ & $0.316^{n s}$ & $0.400^{*}$ & $10.417^{*}$ & $0.014^{*}$ & $0.066^{*}$ & $0.013^{*}$ & $0.006^{*}$ & $0.005^{*}$ \\
\hline Cultivar & & & & & & & & & & & \\
\hline $\begin{array}{c}s \quad X \\
\text { Drought } \\
\text { Levels }\end{array}$ & 8 & $23.522^{*}$ & $27.544^{*}$ & $0.520^{n s}$ & $0.240^{*}$ & $0.714^{n s}$ & $0.002^{*}$ & $0.013^{*}$ & $0.012^{*}$ & $0.002^{n s}$ & $0.004^{*}$ \\
\hline Error & $\begin{array}{l}6 \\
0\end{array}$ & 2.402 & 5.524 & 0.584 & 0.094 & 1.061 & 0.001 & 0.003 & 0.001 & 0.001 & 0.001 \\
\hline Total & $\begin{array}{l}7 \\
4\end{array}$ & & & & & & & & & & \\
\hline
\end{tabular}

Df: degree of freedom and *: significant at $5 \%$. ns: nonsignificant 


\section{Conclusion:}

Water deficit, induced by polyethylene glycol (PEG) affected germination and seedling development. Wheat germination was affected at level at $-0.3 \mathrm{mPa}$. The drought stress significantly reduced the seed germination shoot/root fresh and dry weight. Among cultivars tested T.R. variety is the tolerant one which have the potential to perform well under drought conditions followed by Seri and Condur which exerts sensitivity to water stress conditions at seedling stage parameters. We recommend T.R. variety in any breeding programed to obtains drought tolerant cultivars.

Table 4: Effect of different drought levels on growth parameters of three wheat cultivars

\begin{tabular}{|c|c|c|c|c|c|c|}
\hline parameters & $\begin{array}{c}\text { drought } \\
\text { level }\end{array}$ & Condur & Seri & T.R. & Mean & $L S D$ \\
\hline \multirow{7}{*}{$\begin{array}{c}\text { Shoot } \\
\text { Length }(\mathrm{cm} .) \\
\text { ShL. }\end{array}$} & $0.0 \mathrm{mPa}$ & 14.0468 & 14.6494 & 14.7960 & $14.497 a b$ & \multirow{5}{*}{0.877} \\
\hline & $-0.3 \mathrm{mPa}$ & 15.1666 & 15.4548 & 16.1680 & $15.596 a$ & \\
\hline & $-0.6 \mathrm{mPa}$ & 14.0534 & 13.7412 & 14.2000 & $13.998 \mathrm{~b}$ & \\
\hline & $-0.9 \mathrm{mPa}$ & 12.3134 & 12.0920 & 13.8120 & $12.739 c$ & \\
\hline & $-1.2 m P a$ & 2.3500 & 9.5900 & 12.2884 & $8.076 d$ & \\
\hline & Mean & $11.5860 \mathrm{C}$ & $13.1055 \mathrm{~B}$ & $14.2529 \mathrm{~A}$ & 12.981 & \\
\hline & $L S D$ & \multicolumn{4}{|c|}{1.132} & \\
\hline \multirow{7}{*}{$\begin{array}{c}\text { Root } \\
\text { Length }(\mathrm{cm} .) \\
\text { RL. }\end{array}$} & $0.0 \mathrm{mPa}$ & 9.9934 & 9.9560 & 12.3200 & $10.756 \mathrm{~b}$ & \multirow{5}{*}{1.330} \\
\hline & $-0.3 m P a$ & 12.4002 & 13.0480 & 15.5040 & $13.651 a$ & \\
\hline & $-0.6 \mathrm{mPa}$ & 13.1066 & 12.8600 & 14.9040 & $13.624 a$ & \\
\hline & $-0.9 \mathrm{mPa}$ & 14.5066 & 12.8560 & 17.3096 & $14.891 a$ & \\
\hline & $-1.2 \mathrm{mPa}$ & 2.0500 & 11.8160 & 10.9040 & $8.257 c$ & \\
\hline & Mean & $10.4114 C$ & $12.1072 \mathrm{~B}$ & $14.1883 \mathrm{~A}$ & 12.236 & \\
\hline & $L S D$ & \multicolumn{4}{|c|}{1.717} & \\
\hline \multirow{7}{*}{$\begin{array}{c}\text { Root Number } \\
\text { Rn. }\end{array}$} & $0.0 \mathrm{mPa}$ & 4.4668 & 4.1600 & 4.8800 & $4.502 a$ & \multirow{5}{*}{0.433} \\
\hline & $-0.3 m P a$ & 4.8664 & 3.9600 & 4.7600 & $4.529 a$ & \\
\hline & $-0.6 \mathrm{mPa}$ & 4.4668 & 5.0400 & 5.0400 & $4.849 a$ & \\
\hline & $-0.9 \mathrm{mPa}$ & 4.4668 & 4.7200 & 4.9266 & $4.704 a$ & \\
\hline & $-1.2 \mathrm{mPa}$ & 4.1250 & 4.4934 & 5.0766 & $4.565 a$ & \\
\hline & Mean & $4.4784 B$ & $4.4747 B$ & $4.9366 A$ & 4.630 & \\
\hline & $L S D$ & \multicolumn{4}{|c|}{0.563} & \\
\hline \multirow{7}{*}{$\begin{array}{c}\text { Leaf Number } \\
\text { Ln. }\end{array}$} & $0.0 \mathrm{mPa}$ & 1.6000 & 1.4266 & 1.3600 & $1.462 b c$ & \multirow{5}{*}{0.174} \\
\hline & $-0.3 \mathrm{mPa}$ & 1.8002 & 1.5334 & 1.6400 & $1.658 a b$ & \\
\hline & $-0.6 m P a$ & 1.6668 & 1.8800 & 1.6800 & $1.742 a$ & \\
\hline & $-0.9 \mathrm{mPa}$ & 1.7332 & 1.5600 & 2.0000 & $1.764 a$ & \\
\hline & $-1.2 \mathrm{mPa}$ & 1.2400 & 1.1400 & 1.8400 & $1.407 c$ & \\
\hline & Mean & $1.6080 A B$ & $1.5080 \mathrm{~B}$ & $1.7040 \mathrm{~A}$ & 1.607 & \\
\hline & $L S D$ & \multicolumn{4}{|c|}{0.269} & \\
\hline \multirow{2}{*}{$\begin{array}{c}\text { Leaf Surface } \\
\text { Area }\left(\mathrm{Cm}^{2}\right)\end{array}$} & $0.0 \mathrm{mPa}$ & 3.1734 & 3.1332 & 4.4400 & $3.582 a$ & \multirow{2}{*}{0.583} \\
\hline & $-0.3 m P a$ & 2.5000 & 2.5760 & 3.9700 & $3.015 a b$ & \\
\hline
\end{tabular}

285 
عدد خاص بالأوراق العلمية المقدمة للمؤتمر العلمي الأول للعلوم الزراعية - إنتاج نباتي (5-6 أكتوبر 2019)

\begin{tabular}{|c|c|c|c|c|c|c|}
\hline \multirow[t]{5}{*}{$L S A$} & $-0.6 \mathrm{mPa}$ & 1.6134 & 2.5500 & 3.5000 & $2.554 b c$ & \\
\hline & $-0.9 m P a$ & 1.4800 & 1.9874 & 2.3200 & $1.929 \mathrm{~cd}$ & \\
\hline & $-1.2 \mathrm{mPa}$ & 0.4000 & 1.9000 & 2.1800 & $1.493 d$ & \\
\hline & Mean & $1.8334 C$ & $2.4293 B$ & $3.2820 \mathrm{~A}$ & 2.515 & \\
\hline & $\angle S D$ & \multicolumn{4}{|c|}{0.783} & \\
\hline \multirow{7}{*}{$\begin{array}{c}\text { Seedling } \\
\text { Fresh } \\
\text { Weight } \\
\text { SFW. }\end{array}$} & $0.0 \mathrm{mPa}$ & 0.1426 & 0.1172 & 0.1776 & $0.146 a$ & \multirow{5}{*}{0.0142} \\
\hline & $-0.3 \mathrm{mPa}$ & 0.1208 & 0.1080 & 0.1176 & $0.115 b$ & \\
\hline & $-0.6 m P a$ & 0.0954 & 0.1056 & 0.1138 & $0.105 b c$ & \\
\hline & $-0.9 \mathrm{mPa}$ & 0.0936 & 0.0712 & 0.1076 & $0.091 c$ & \\
\hline & $-1.2 \mathrm{mPa}$ & 0.0262 & 0.0634 & 0.0982 & $0.063 d$ & \\
\hline & Mean & $0.0957 B$ & $0.0931 B$ & $0.1230 \mathrm{~A}$ & 0.104 & \\
\hline & $L S D$ & \multicolumn{4}{|c|}{0.0183} & \\
\hline \multirow{7}{*}{$\begin{array}{c}\text { Shoot Fresh } \\
\text { Weight } \\
\text { ShFW }\end{array}$} & $0.0 \mathrm{mPa}$ & 0.2760 & 0.0816 & 0.5220 & $0.293 a$ & \multirow{5}{*}{0.029} \\
\hline & $-0.3 m P a$ & 0.2538 & 0.0664 & 0.4200 & $0.247 b$ & \\
\hline & $-0.6 \mathrm{mPa}$ & 0.2058 & 0.0632 & 0.3680 & $0.212 b c$ & \\
\hline & $-0.9 m P a$ & 0.1620 & 0.0508 & 0.3420 & $0.185 c$ & \\
\hline & $-1.2 \mathrm{mPa}$ & 0.0140 & 0.0400 & 0.2980 & $0.117 d$ & \\
\hline & Mean & $0.1823 B$ & $0.0604 \mathrm{C}$ & $0.3900 \mathrm{~A}$ & 0.211 & \\
\hline & $L S D$ & \multicolumn{4}{|c|}{0.037} & \\
\hline \multirow{7}{*}{$\begin{array}{c}\text { Shoot Dry } \\
\text { Weight } \\
\text { ShDW }\end{array}$} & $0.0 \mathrm{mPa}$ & 0.0180 & 0.0322 & 0.2440 & $0.098 a$ & \multirow{5}{*}{0.0202} \\
\hline & $-0.3 \mathrm{mPa}$ & 0.0220 & 0.0230 & 0.1400 & $0.062 b$ & \\
\hline & $-0.6 m P a$ & 0.0196 & 0.0240 & 0.0540 & $0.033 c$ & \\
\hline & $-0.9 m P a$ & 0.0198 & 0.0308 & 0.0500 & $0.034 c$ & \\
\hline & $-1.2 \mathrm{mPa}$ & 0.0100 & 0.0228 & 0.0478 & $0.027 c$ & \\
\hline & Mean & $0.0179 B$ & $0.0266 B$ & $0.1072 \mathrm{~A}$ & 0.051 & \\
\hline & $L S D$ & \multicolumn{4}{|c|}{0.0266} & \\
\hline \multirow{7}{*}{$\begin{array}{c}\text { Root Fresh } \\
\text { Weight } \\
\text { RFW }\end{array}$} & $0.0 \mathrm{mPa}$ & 0.0662 & 0.0860 & 0.1620 & $0.105 a$ & \multirow{5}{*}{0.0190} \\
\hline & $-0.3 m P a$ & 0.0428 & 0.0860 & 0.1020 & $0.077 b c$ & \\
\hline & $-0.6 m P a$ & 0.0500 & 0.1240 & 0.1100 & $0.095 a b$ & \\
\hline & $-0.9 m P a$ & 0.0400 & 0.0760 & 0.0840 & $0.067 c$ & \\
\hline & $-1.2 m P a$ & 0.0350 & 0.0620 & 0.0760 & $0.058 c$ & \\
\hline & Mean & $0.0468 \mathrm{C}$ & $0.0868 B$ & $0.1068 \mathrm{~A}$ & 0.080 & \\
\hline & $L S D$ & \multicolumn{4}{|c|}{0.0246} & \\
\hline \multirow{5}{*}{$\begin{array}{c}\text { Root Dry } \\
\text { Weight } \\
\text { RDW }\end{array}$} & $0.0 \mathrm{mPa}$ & 0.0222 & 0.0088 & 0.1360 & $0.056 a$ & \multirow{5}{*}{0.0140} \\
\hline & $-0.3 m P a$ & 0.0200 & 0.0132 & 0.0460 & $0.026 b$ & \\
\hline & $-0.6 m P a$ & 0.0164 & 0.0220 & 0.0280 & $0.022 b$ & \\
\hline & $-0.9 m P a$ & 0.0084 & 0.0114 & 0.0260 & $0.015 b$ & \\
\hline & $-1.2 m P a$ & 0.0100 & 0.0092 & 0.0140 & $0.011 b$ & \\
\hline & Mean & $0.0154 B$ & $0.0129 B$ & $0.0500 \mathrm{~A}$ & 0.026 & \\
\hline
\end{tabular}

Values in mean column sharing same letter are statistically no-significant at $5 \%$. 


\section{References:}

Ahmad, M., G. Shabbir, M.M. Nasir, and M. K. N. Shah. 2013. Identification of Drought Tolerant Wheat Genotypes Based on Seedling Traits. Sarhad J. Agric. 29(1): 21-27.

Almaghrabi, Omar A. 2012. Impact of Drought Stress on Germination and Seedling Growth Parameters of Some Wheat Cultivars. Life Science Journal 9(1): 590-598.

Armin, M., M. Asgharipour, and M. Razavi-Omrani. 2010. The Effect of Seed Priming on Germination and Seedling Growth of Watermelon (Citrullus Lanatus). Adv. Environ. Biol. 4(3): 501-505.

Ashraf, M., and S. Mehmood. 1990. Response of Four Brassica Species to Drought Stress. Environ. Expt. Bot. 30: 93-100.

Association of Official Seed Analysis (AOSA). 1983. "Seed Vigor Testing Handbook. Contibution" No.32 to the Handbook on Seed Testing.

Bahieldin, A. et al. 2005. Field Evaluation of Transgenic Wheat Plants Stably Expressing the HVA1 Gene for Drought Tolerance. Physiologia Plantarum 123(4): 421-427.

Bayoumi, T.Y., M. H. Eid, and E.M. Metwali. 2008. Application of Physiological and Biochemical Indices as a Screening Technique for Drought Tolerance in Wheat Genotypes. African Journal of Biotechnology 7(14): 2341-2352.

Chachar, M.H. et al. 2014. In-Vitro Screening Technique for Drought Tolerance of Wheat ( Triticum Aestivium L .) Genotypes at Early Seedling Stage. Journal of Agricultural Technology 10(6): 1439-1450.

Chachar, N.A. et al. 2014. Exploration of Genetic Diversity between S1x Wheat Genotypes for Drought Tolerance. Climate Change outlook and adaptation 2(1): 27-33.

Chachar, Z. et al. 2016. Identification of Drought Tolerant Wheat Genotypes Under Water Deficit Conditions. International Journal of Research 44(22): 206-214.

Dhanda, S.S., G.S. Sethi, and R.K. Behl. 2004. Indices of Drought Tolerance in Wheat Genotypes at Early Stages of Plant Growth. J. Agron. Crop Sci. 190: 1-6.

Dodd, G.L., and L.A. Donovan. 1999. Water Potential and Ionic Effects on Germination and Seedling Growth of Two Cold Desert Shrubs. Am. J. Bot. 86: 1146-1153.

Emmerich, W. E., and S. P. Hardegree. 1991. Seed Germination in Polyethylene Glycol Solution: Effects of Filter Paper Exclusion and Water Vapor Loss. Crop Science 31(2): 454-458.

Fraser, T. E., W. K. Silk, and T. L. Rost. 1990. Effects of Low Water Potential on Cortical 287

http://www.misuratau.edu.ly/journal/jmuas/ 2019 المجلد الأول العدد الأول ديسمبر 
عدد خاص بالأور اق العلمية المقدمة للمؤتمر العلمي الأول للعلوم الزعلة الزراعية ــ إنتاج نباتي (5-6 أكتوبر 2019)

Cell Length in Growing Regions of Maize Roots. Plant Physiology 93(2): 648-651.

Giri, G.S., and W.F. Schillinger. 2003. Seed Priming Winter Wheat for Germination, Emergence and Yield. Crop Sci. 43: 2135-2141.

Heikal, M.M., M.A. Shaddad, and A.M. Ahmed. 1981. Effect of Water Stress and Gibberellic Acid on Germination of Flax, Sesame and Onion Seed. Biological Plantarum 24(2): 124-129.

ISTA, Association, International Seed Testing. 1993. "Hand Book for Seedling Evaluation". Zurich, Switzerland.

Janmohammadi, M.P. Moradi Dezfuli, and F. Sharifzadeh. 2008. Seed Invigoration Techniques to Improve Germination and Early Growth of Inbred Line of Maize under Salinity and Drought Stress. Gen. Appl. Plant Phys. 34(Special Issue,(3-4)): 215-226.

Jing, R.L., and X.P. Chang. 2003. Genetic Diversity in Wheat (Triticum Aestivum L.) Germplasm Resources with Drought Resistance. Acta Botanica Boreal-Occident Sinica 23: 410-416.

Kamran, M., M. Shahbaz, M. Ashraf, and A. A. Nudrat. 2009. Alleviation of DroughtInduced Adverse Effects in Spring Wheat (Triticum Aestivum L.) Using Proline as a PreSowing Seed Treatment. Pakistan Journal of Botany 41(2): 621-632.

Kaufmann, M.R, and A.N Eckard. 1971. Evaluation of Water Stress Control with Polyethylene Glycols by Analysis of Guttation. Plant physiology 47(4): 453-456.

Khakwani, A., M.D. Dennett, and M. Munir. 2011. Early Growth Response of Six Wheat Varieties Under Artificial Osmotic Stress Condition. Pak. J. Agri. Sci 48(2): 119-123.

Khan, N., and F.N. Naqvi. 2011. Heritability of Morphological Traits in Bread Wheat Advanced Lines Under Irrigated and Non-Irrigated Conditions. Asian J. Agric. Sci. 3(3): 215-222.

Khayatnezhad, M., R. Gholamin, and S. Jamaati-e-somarin. 2010. Effects of Peg Stress on Corn Cultivars ( Zea Mays L .) At Germination Stage. Applied Sciences 11(5): 504506.

Kulkarni, M., and U. Deshpande. 2007. In-Vitro Screnning of Tomato Genotypes for Drought Resistance Using Polyethylene Glycol. Afr. J. Biotechnology 6: 691-696.

Kumar, R.R., G.R. Naik, and K. Karjol. 2011.Variation of Sensitivity to Drought Stress in Pigeon Pea (Cajanus Cajan Millsp) Cultivars during Seed Germination and Early Seedling GrowtH. World Journal of Science and Technology 1(1): 11-18.

Lu, Z., and P.M. Neumann. 1998.Water-Stressed Maize, Barley and Rice Seedlings Show 288 
Species Diversity in Mechanisms of Leaf Growth Inhibition. J.Exp. Bot. 49: 1945-1952.

Metwali, M.R.E., H.E. Manal, and Y.B. Tarek. 2011. Agronomical Traits and Biochemical Genetic Markers Associated with Salt Tolerance in Wheat Cultivars (Triticum Aestivum L.). Australian J. of Basic and Applied science 5(5): 174-183.

Michel, B.E., and M. R. Kaufmann. 1973. The Osmotic Potential of Polyethylene Glycol 60001. Plant Physiol 51: 914-916.

Money, N.P. 1989. Osmotic Pressure of Aqueous Polyethylene Glycols. Relationship between Molecular Weight and Vapor Pressure Deficit. Plant Physiol. 91: 766-769.

Pan, X.Y., Y.F. Wang, G.X. Wang, Q.D. Cao, and J. Wang. 2002. Relationship between Growth Redundancy and Size Inequality in Spring Wheat Populations Mulched with Clear Plastic Film. Acta Phytoecol. Sinica 26: 177-184.

Pessarakli, M. 1999. "Handbook of Plant and Crop Stress". 2nd Ed.,. New York: Marcel Dekker Inc.,.

Ranal, M. A., D. G. DeSantana, W. R. Ferreira, and C. Mendes-Rodrigues. 2009. Calculating Germination Measurements and Organizing Spreadsheets. Revista Brasileira de Botânica 32(4): 849-855.

Ranal, M.A., and D.G. De Santana. 2006. How and Why to Measure the Germination Process?. Revista Brasileira de Botânica 29(1): 1-11.

Sadeghi, H., K. Fardin, Y. Liela, and S. Saman. 2011. Effect of Seed Osmopriming on Seed Germination Behavior and Vigor of Soybean (Glycin Max L.). ARPN Journal of Agricultural and Biological Science 6(1): 39-43.

Scott, S. J., R. A. Jones, and W. A. Williams. 1984. Review of Data Analysis Methods for Seed Germination. Crop Sci. 24(24): 1192-1199.

Shahryari, R., and V. Mollasadeghi. 2011. Introduction of Two Principle Components for Screening of Wheat Genotypes under End Seasonal Drought. Adv. Environ. Biol. 5(3): $519-522$.

Shao, H.B., L.Y.Chu, C.A. Jaleel, P. Manivannan, R. Panneerselvam, and M.A. Shao. 2009. Understanding Water Deficit Stress-Induced Changes in the Basic Metabolism of Higher Plants Biotechnologically and Sustainably Improving Agriculture and the Eco Environment in Arid Regions of the Globe. Crit. Rev. Biotechnol 29: 131-151.

Stell, R.G., and J.H. Torrie. 1980. "Principles and Procedure of Statistics. A Biometrical Approach". 2nd Inter. New York. USA: Tokyo Mc graw Hill, Book Co.

Steven, R.B., P.F. Byrne, S.D. Reid, W.L.Bauerle, J.K. McKay, and S.D.Haley. 2016. 289 
Root Traits Contributing to Drought Tolerance of Synthetic Hexaploid Wheat in a Greenhouse Study. Euphytica 207(1): 213-224.

Taregh, G., S. Mostafa, valizadeh, and S. Hossein. 2011. Effect of Drought on Germination Indices and Seedling Growth of 12 Bread Wheat Genotypes . Advances In Environmental Biology 5(6): 1034-1039.

Wang, X.Y., P. Vinocur, and A. Altman. 2003. Plant Responses to Drought, Salinity and Extreme Temperatures: Towards Genetic Engineering for Stress Tolerance. Planta 218: $1-14$.

Zhu,J. 2006. Effects of Drought Stresses Induced by Polyethylene Glycol on Germination of Pinus Sylvestris Var. Mongolica Seeds from Pollination Forests on Sandy Land. Natural and Polination Forests on Sandy. Land Journal of Forest Research 11(5): 319328.

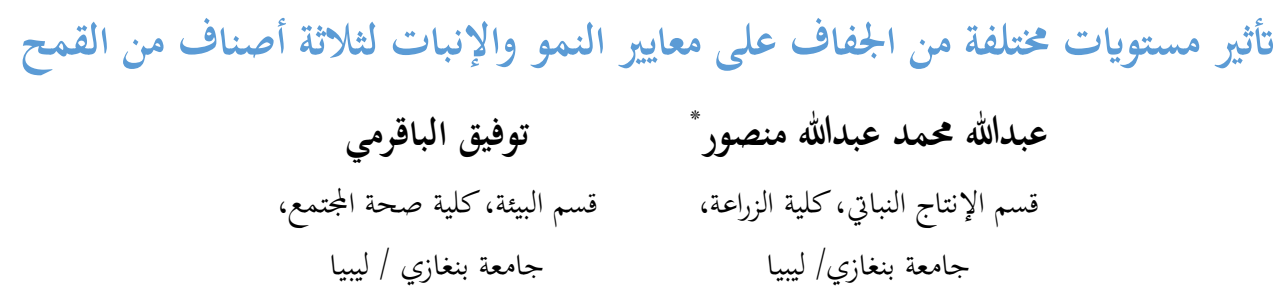

*Abdalla.mansour@uob.edu.ly

https://doi.org/10.36602/jmuas.2019.v01.01.22

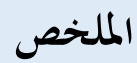

تم تقييم إنبات ثلاثة أصناف من القمح (Triticum aestivum L.) وهي CONDOR و CORI

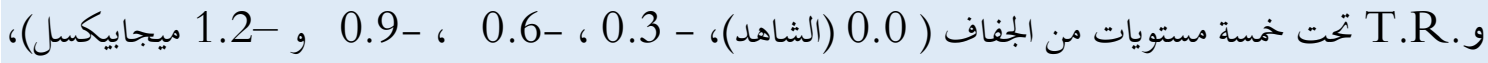

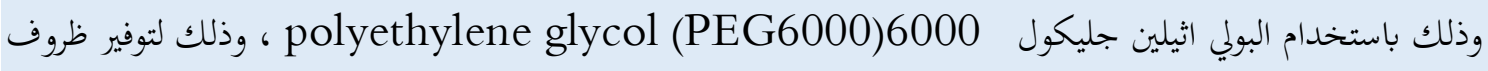

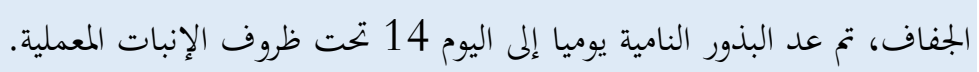
تم أخذ القياسات لعملية الإنبات بقياس النسبة المئوية للإنبات ومتوسط زمن الإنبات ومعامل الإنبات ومتوسط الإنبات سمعليه سرعة الإنبات، كما تم قياس الطول والوزن الرطب والجاف لكل من المجموع الخضري والمجموع الجذري وللنبتة كاملة، إضافة إلى لئل

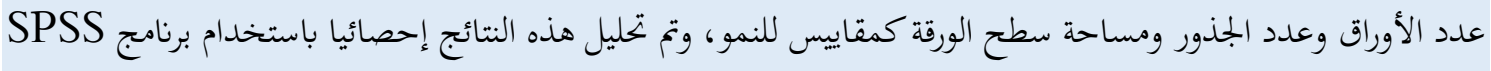
لتحديد الفروق المعنوية بين الأصناف المدروسة وكذلك المعالجات المدات المستخدمة.

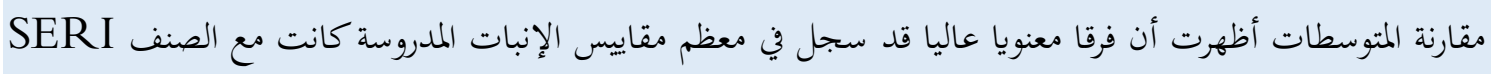

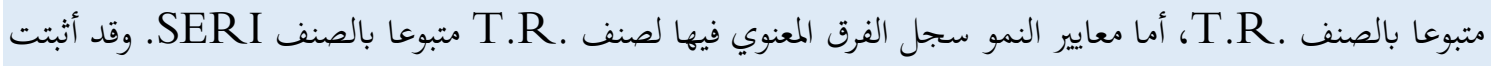
النتائج أن بذور الأصناف الثلاثة أنبتت بشكل جيد عند المستويات المنخفضة من البولي اثيلين جليكول 6000 والذي أثر على كل مقاييس الإنبات والنمو. 